\title{
Fault detection and isolation for systems defined over graphs
}

\author{
P. Rapisarda, A.R.F. Everts, and M.K. Camlibel
}

\begin{abstract}
We consider the problem of fault detection and isolation for a class of linear dynamical systems defined by a graph containing faulty vertices and observer vertices. Using a geometric approach, we provide a characterization of the smallest conditioned invariant subspaces generated by faults in terms of the underlying graph structure. Based on this characterization, we give graph-theoretic conditions guaranteeing fault detectability. In addition, we provide a condition under which fault detectability fails.
\end{abstract}

\section{INTRODUCTION}

Detecting and identifying faults in multi-agent systems is particularly relevant, given the absence of a centralized observer monitoring the whole network and the practical applications of such systems, where faults either in the agents or in the communication structure can have serious consequences. This leads naturally to considering design issues; among these the establishment of topologies and the organization of communication structures that guarantee prompt detection and accurate isolation of faults are of primary importance.

In the literature on multi-agent systems within the control community, several approaches have been proposed for fault detection and isolation. Among these, those closer to the results presented here are [3], [6], [7]. In these references the geometric approach to fault detection and isolation based on unknown input observers pioneered in [4], [5] is used. In [3], [7] problems of leader collocation to guarantee controllability of a multi-agent system under communication failure is studied; in [6] the authors study the problem of reliable computation in consensus networks.

In this paper, we consider a class of linear dynamical systems defined by a undirected graph containing faulty vertices and observer vertices. Two disjoint sets of agents are identified in the network: those prone to failure (called "faulty") and those whose output is measurable (called "observer"). Faults such as total communication failures, biased sensing, etc. can be modelled in a straightforward way in our framework. Fault detection is performed by an unknown input observer, and stated in the geometric language of [5], i.e. output separability of fault subspaces.

First we present a characterization of the smallest conditioned invariant subspaces that are generated by the faults.

P. Rapisarda is with the Vision, Learning and Control group, School of Electronics and Computer Science, University of Southampton, UK, e-mail: pr3eecs.soton.ac.uk

A.R.F. Everts and M.K. Çamlibel are with the Johann Bernoulli Institute for Mathematics and Computer Science, University of Groningen, P.O. Box 407, 9700 AK, the Netherlands, e-mail: a.r.f.everts@rug.nl, m.k. camlibel@rug.nl

The work of the second author is funded by NWO (The Netherlands Organisation for Scientific Research) project CODAVI (613.001.108).
This characterization is exploited in order to give graph topological conditions guaranteeing output separability in terms of distances between faulty agents and observer ones. In addition, we study the case where two faulty vertices share exactly the same neighbors in order to present a condition under which fault detectability fails.

The organization of the paper is as follows. In section II we review concepts and basic results of geometric control theory for the sake of completeness. This section also introduces graph theoretical tools that will be used later on in the paper. Section III discusses the problem of fault detection in a geometric setting. In section IV, we present main results of the paper. Finally, the paper closes with the conclusions in section $\mathrm{V}$.

\section{BACKGROUND MATERIAL}

\section{A. Geometric control theory}

Geometric control theory, illustrated in depth in e.g. [1] and [8], plays an important role in this paper. We briefly review the definitions and results playing a role in the rest of the paper.

Given a linear, time-invariant system

$$
\begin{aligned}
& \dot{x}=A x+B u \\
& y=C x
\end{aligned}
$$

where $A \in \mathbb{R}^{n \times n}, B \in \mathbb{R}^{n \times m}, C \in \mathbb{R}^{p \times n}$, a subspace $\mathcal{S} \subseteq$ $\mathbb{R}^{n}$ is called $(C, A)$-invariant (equivalently, conditioned invariant) if

$$
A(\mathcal{S} \cap \operatorname{ker}(C)) \subseteq \mathcal{S}
$$

This condition is equivalent to the existence of a $K \in \mathbb{R}^{n \times p}$ such that $\mathcal{S}$ is $(A+K C)$-invariant, i.e.

$$
(A+K C) \mathcal{S} \subseteq \mathcal{S} .
$$

Such a matrix $K$ is called a friend of $\mathcal{S}$. The set consisting of all friends of a $(C, A)$-invariant subspace $\mathcal{S}$ is denoted by

$$
\mathcal{K}(\mathcal{S}):=\left\{K \in \mathbb{R}^{n \times p} \mid(A+K C) \mathcal{S} \subseteq \mathcal{S}\right\}
$$

A family $\left\{\mathcal{S}_{i}\right\}_{i=1}^{k}$ of $(C, A)$-invariant subspaces of $\mathbb{R}^{n}$ is called compatible if the subspaces $\mathcal{S}_{i}$ have a common friend, i.e. if there exists $K \in \mathbb{R}^{n \times p}$ such that $K \in \mathcal{K}\left(\mathcal{S}_{i}\right)$ for all $i=1,2, \ldots, k$, equivalently if

$$
\bigcap_{i=1}^{k} \mathcal{K}\left(\mathcal{S}_{i}\right) \neq \varnothing .
$$


Given the dynamics (1), a family of subspaces $\left\{\mathcal{S}_{i}\right\}_{i=1}^{k}, S_{i} \subset$ $\mathbb{R}^{n}$ is called output separable (see [5]) if

$$
C \mathcal{S}_{i} \cap\left(\sum_{j \neq i} C \mathcal{S}_{j}\right)=\{0\} .
$$

Output separable $(C, A)$-invariant subspaces admit a common friend.

Proposition 1 Let $\left\{\mathcal{S}_{i}\right\}_{i=1}^{k}$ be a family of $(C, A)$-invariant subspaces. If $\left\{\mathcal{S}_{i}\right\}_{i=1}^{k}$ is output separable, then there exists $K \in \mathbb{R}^{n \times p}$ such that $(A+K C) \mathcal{S}_{i} \subseteq \mathcal{S}_{i}$ for all $i=$ $1,2, \ldots, k$.

Proof: The proof is analogous to that of Lemma 2 on p. 841 of [5] and is omitted.

Given a subspace $\mathcal{M} \subset \mathbb{R}^{n}$, in the following the set of all conditioned invariant subspaces containing $\mathcal{M}$ is denoted by $\mathfrak{S}(\mathcal{M})$. Since $\mathfrak{S}(\mathcal{M})$ is closed under intersection, it admits a unique smallest (in the inclusion order) element, which in the following we denote by $\mathcal{S}^{*}(\mathcal{M})$. Whenever it is clear from the context which the subspace $\mathcal{M}$ is in use, we simply write $\mathcal{S}^{*}$.

Such a minimal subspace can be computed by the following subspace algorithm (see e.g. Algorithm 4.1.1 p. 203 of [1]):

$$
\begin{aligned}
& \mathcal{S}^{0}:=\mathcal{M} \\
& \mathcal{S}^{k}:=\mathcal{M}+A\left(\mathcal{S}^{k-1} \cap \operatorname{ker} C\right)
\end{aligned}
$$

for $k=1,2, \ldots$. As these subspaces are nested, that is

$$
\mathcal{S}^{k} \subseteq \mathcal{S}^{k+1},
$$

existence of an integer $\ell$ such that $0 \leqslant \ell \leqslant n$ and

$$
\mathcal{S}^{\ell}=\mathcal{S}^{\ell+1}
$$

readily follows. It is well-known that

$$
\mathcal{S}^{*}=\mathcal{S}^{\ell} .
$$

\section{B. Graphs and zero patterns}

Consider a simple graph $G=(V, E)$, i.e. undirected and unweighted graph containing no multiple edges or loops on vertices. Let the vertex set $V$ be given by

$$
V=\{1,2, \ldots, n\}
$$

and the edge set $E$ a subset of all unordered pairs of vertices, that is $E \subseteq\{\{i, j\} \mid i, j \in V\}$.

Associated to such a graph $G=(V, E)$, we define a family of matrices, called the qualitative class of $G$, by

$$
\mathcal{Q}(G)=\left\{X \in \mathbb{R}^{n \times n} \mid \text { for } i \neq j, X_{i j} \neq 0 \Leftrightarrow\{i, j\} \in E\right\} .
$$

Typical elements of the qualitative class are adjacency, Laplacian and normalized Laplacian matrices corresponding to a simple graph. Note that the elements of the qualitative class may correspond to matrices associated to a weighted graph, even though the graph $G$ is itself simple and hence unweighted.
We say that a graph $H=(V, E)$ is bipartite if there exist disjoint vertex sets $V^{-}$and $V^{+}$such that $V^{-} \cup V^{+}=V$ and the edge set $E$ contains only edges connecting one vertex from $V^{-}$and the other from $V^{+}$. With a slight abuse of notation, we write $H=\left(V^{-}, V^{+}, E\right)$ for bipartite graphs.

Associated to a bipartite graph $H=\left(V^{-}, V^{+}, E\right)$ with

$$
\begin{aligned}
& V^{-}=\left\{r_{1}, r_{2}, \ldots, r_{p}\right\} \\
& V^{+}=\left\{c_{1}, c_{2}, \ldots, c_{q}\right\},
\end{aligned}
$$

we define a pattern class by

$$
\mathcal{P}(H):=\left\{Y \in \mathbb{R}^{p \times q} \mid Y_{i j} \neq 0 \Leftrightarrow\left\{r_{i}, c_{j}\right\} \in E\right\} .
$$

Note that the matrices in the pattern class are not necessarily square unlike those in the qualitative class.

A set of $t$ edges of a bipartite graph $H=\left(V^{-}, V^{+}, E\right)$ that do not share a common vertex is called a $t$-matching. A $t$-matching is called constrained if there is no other $t$ matching between the matched vertices.

The following is a classical result (see [2, Thm. 3.9]) in the study of the minimal rank of a given pattern class.

Theorem 2 Let $H=\left(V^{-}, V^{+}, E\right)$ be a bipartite graph with $V^{-}=\left\{r_{1}, r_{2}, \ldots, r_{p}\right\}$ and $V^{+}=\left\{c_{1}, c_{2}, \ldots, c_{q}\right\}$. All matrices $Y \in \mathcal{P}(H)$ are of full rank if and only if $H$ admits a constrained $\min (p, q)$-matching.

\section{Problem Statement}

Let $G=(V, E)$ be a simple and connected graph with the vertex set

$$
V=\{1,2, \ldots, n\} .
$$

Two subsets of $V$ will play an important role in the sequel. We denote these sets by $V_{F}$ (faulty vertices) and $V_{O}$ (observer vertices). For simplicity, we assume that these sets are disjoint and that the first $q$ vertices are faulty and the last $s$ are observer, that is

$$
\begin{aligned}
& V_{F}=\{1,2, \ldots, q\} \\
& V_{O}=\{n-s+1, n-s+2, \ldots, n\} .
\end{aligned}
$$

Throughout the paper, we will consider systems of the form

$$
\begin{aligned}
& \dot{x}=X x+M f \\
& y=N x
\end{aligned}
$$

where $x$ is the state, $f$ is the fault mode and $y$ is the output vector. The matrices $X, M$ and $N$ are related to the given simple graph $G$ and the pair $\left(V_{F}, V_{O}\right)$ in the sense that $X$ belongs to $\mathcal{Q}(G), M$ encodes the faulty vertices and $N$ the observer vertices, that is

$$
M=\left[\begin{array}{c}
I_{q} \\
0_{n-q, q}
\end{array}\right] \text { and } N=\left[\begin{array}{ll}
0_{s, n-s} & I_{s}
\end{array}\right] .
$$

The problem we will address in this paper amounts to setting up an observer

$$
\begin{aligned}
& \dot{\hat{x}}=(X+K N) \widehat{x}-K y \\
& \widehat{y}=N \widehat{x}
\end{aligned}
$$


where $K \in \mathbb{R}^{n \times p}$, in order to detect if and which faults are active.

To elaborate on what we mean by detecting faults, define the error by

$$
e:=\widehat{x}-x
$$

and note that it satisfies the dynamics

$$
\begin{aligned}
\dot{e} & =(X+K N) e-M f \\
r & =N e
\end{aligned}
$$

where $r$ is the innovation (sometimes also called residual) term.

Assuming that $e(0)=0$, if only the $i$-th fault occurs, i.e., if $f_{i} \neq 0$ and $f_{j}=0$ for $j \neq i$, the error in (7) is confined to the smallest $(X+K N)$-invariant subspace containing $\operatorname{im} M_{i}$, where $M_{i}$ denotes the $i$ th column of the matrix $M$. Under such strong assumption (implying that the observer is initialized precisely with the same initial conditions as the system), the fault detection and isolation problem can be stated in the geometric language illustrated in sect. II as follows:

Given the dynamics (5), find a family $\left\{\mathcal{S}_{i}\right\}_{i=1}^{q}$ of subspaces of $\mathbb{R}^{n}$ and associated $K \in \mathbb{R}^{n \times p}$ such that

C1) $(X+K N) \mathcal{S}_{i} \subseteq \mathcal{S}_{i}, i=1, \ldots, q$,

C2) $\operatorname{im} M_{i} \subseteq \mathcal{S}_{i}, i=1, \ldots, q$,

C3) $N \mathcal{S}_{i} \cap\left(\sum_{j \neq i} N \mathcal{S}_{j}\right)=\{0\}$.

If such a family of subspaces exists, $e(0)=0$, and only the $i$-th fault occurs, then it follows from the structure of the observer (6) that $e(t)$ belongs to $\mathcal{S}_{i}$ for all $t \in \mathbb{R}$ (condition $\mathrm{C} 1$ and $\mathrm{C} 2$ ). Because of output separability (condition C3), in case of multiple faults, information on the presence and type of faults can be obtained by projecting the innovation vector $r$ on $N \mathcal{S}_{i}$ for $i=1,2, \ldots, q$.

In the generic case of $e(0) \neq 0$, the asymptotic stability of the observer comes into play, and the common friend $K$ of the family $\left\{\mathcal{S}_{i}\right\}$ must be such that

$$
\lim _{t \rightarrow \infty} e(t)=0
$$

i.e. the conditions $\mathrm{C} 1-\mathrm{C} 3$ must be supplemented by the condition

$$
\text { C4) } \sigma(X+K N) \subset \mathbb{C}_{-} \text {. }
$$

The main goal of this paper is to investigate graph topological sufficient conditions that enable fault detection and isolation as explained above.

\section{OUTPUT SEPARABILITY: GRAPH-THEORETIC CONDITIONS}

Given particular choices of $X, M$ and $N$ in (5), checking for the existence of an output separable family $\left\{\mathcal{S}_{i}\right\}_{i=1}^{q}$ that satisfies conditions $\mathrm{C} 1-\mathrm{C} 3$ is a straightforward linear algebra problem. For this, we define $\mathcal{S}_{i}^{*}$ to be the smallest $(N, X)$-invariant subspace containing $\operatorname{im} M_{i}$ for $i=$ $1,2, \ldots, q$. Then the following result holds.
Lemma 3 Consider the system (5). There exists a family $\left\{\mathcal{S}_{i}\right\}_{i=1}^{q}$ of subspaces satisfying conditions $C 1-C 3$ if and only if $\left\{\mathcal{S}_{i}^{*}\right\}_{i=1}^{q}$ is output separable.

Proof: See the proof of Theorem 3 p. 841 of [5].

Thus in order to check whether the FDI approach of section III can be applied to a given network, one needs to compute the $(N, X)$-invariant subspaces $\mathcal{S}_{i}^{*}$ via the algorithm (4), and then check the output separability of $\left\{\mathcal{S}_{i}^{*}\right\}_{i=1}^{q}$. Necessary and sufficient conditions for the existence of a stabilizing common friend of subspaces $\mathcal{S}_{i}$ are given in Theorems 9 and 10 of [5].

In this section we pursue another line of thought, providing a sufficient condition for output separability based on graph-topological considerations, as in [3], [7]. Such approach has the advantage of avoiding potential numerical and computational complexity issues associated with linear algebra computations for large-scale networks, providing instead robust conditions based on discrete mathematics. Moreover, it offers insight into the structural properties of networks, with potentially useful applications for example in the design of network systems.

Moreover, the conditions we will provide are going to be valid not only for a particular choice of the matrix $X$ but rather for a family of matrices within the qualitative class, namely the so-called distance-information preserving matrices.

To elaborate on this class of matrices, recall that for a graph $G=(V, E)$ the distance between two vertices is the length of the shortest path connecting them. The distance between the vertices $i$ and $j$ is denoted by $\operatorname{dist}(i, j)$. By convention, $\operatorname{dist}(i, j):=\infty$ if no path exists between vertex $i$ and vertex $j$, and $\operatorname{dist}(i, i)=0$ for any vertex $i$.

Definition 4 A matrix $X \in \mathbb{R}^{n \times n}$ is distance-information preserving with respect to the graph $G=(V, E)$ if

$$
\left(X^{k}\right)_{i, j} \begin{cases}=0 & \text { if } \operatorname{dist}(i, j)>k \\ \neq 0 & \text { if } \operatorname{dist}(i, j)=k\end{cases}
$$

for $k \geqslant 0$.

Clearly, every distance-information preserving matrix belongs to the qualitative class $\mathcal{Q}(G)$ but converse is not true in general. Laplacian and adjacency matrices, frequently used in describing graph structures, are typical instances of distanceinformation preserving matrices.

Furthermore, we define the distance of a vertex $i \in V$ from a nonempty subset of vertices $V^{\prime} \subseteq V$ as follows:

$$
\operatorname{dist}\left(i, V^{\prime}\right):=\min _{j \in V^{\prime}} \operatorname{dist}(i, j) \text {. }
$$

We begin with presenting a characterization of the subspaces $\mathcal{S}_{i}^{*}$.

Lemma 5 Consider the system (5). Suppose that $X$ is a distance-information preserving matrix with respect to the 
graph $G=(V, E)$. Let $i \in V_{F}$ and $d_{i}=\operatorname{dist}\left(i, V_{O}\right)$. Then, we have

$$
\begin{aligned}
\mathcal{S}_{i}^{*} & =\operatorname{im}\left[\begin{array}{llll}
M_{i} & X_{i} & \cdots & \left(X^{d_{i}}\right)_{i}
\end{array}\right] \\
N \mathcal{S}_{i}^{*} & =\operatorname{im} N\left(X^{d_{i}}\right)_{i}
\end{aligned}
$$

where $X_{i}$ denotes the ith column of the matrix $X$.

Proof: We prove the statement by employing the recursion (4), starting with

$$
\mathcal{S}^{0}=\operatorname{im} M_{i}
$$

Since $V_{F} \cap V_{O}=\varnothing$, it follows that

$$
\operatorname{im} M_{i} \subseteq \operatorname{ker} N \text {. }
$$

Thus, we obtain

$$
\begin{aligned}
\mathcal{S}^{1} & =\operatorname{im} M_{i}+X\left(\mathcal{S}^{0} \cap \operatorname{ker} N\right) \\
& =\operatorname{im} M_{i}+X\left(\operatorname{im} M_{i} \cap \operatorname{ker} N\right) \\
& \stackrel{(9)}{=} \operatorname{im~} M_{i}+X \operatorname{im} M_{i} .
\end{aligned}
$$

Note that $X \operatorname{im} M_{i}=\operatorname{im} X_{i}$ by the definition of $M$. Hence, we get

$$
\mathcal{S}^{1}=\operatorname{im} M_{i}+\operatorname{im} X_{i} .
$$

Since $V_{F} \cap V_{O}=\varnothing$, we have $d_{i}>0$. Together with the connectedness of $G$ this implies that $\mathcal{S}^{1}$ is strictly larger than $\mathcal{S}^{0}$.

Next, we note that

$$
\begin{aligned}
\mathcal{S}^{2} & =\operatorname{im} M_{i}+X\left(\mathcal{S}^{1} \cap \operatorname{ker} N\right) \\
& =\operatorname{im} M_{i}+X\left(\left(\operatorname{im} M_{i}+\operatorname{im} X_{i}\right) \cap \operatorname{ker} N\right) .
\end{aligned}
$$

It follows from (9) that

$$
\begin{aligned}
\left(\operatorname{im} M_{i}\right. & \left.+\operatorname{im} X_{i}\right) \cap \operatorname{ker} N \\
& =\left(\operatorname{im} M_{i} \cap \operatorname{ker} N\right)+\left(\operatorname{im} X_{i} \cap \operatorname{ker} N\right) \\
& =\operatorname{im} M_{i}+\left(\operatorname{im} X_{i} \cap \operatorname{ker} N\right) .
\end{aligned}
$$

Now, we distinguish two cases: $\operatorname{dist}\left(i, V_{O}\right)=1$ and $\operatorname{dist}\left(i, V_{O}\right)>1$. In the first case, we have

$$
\operatorname{im} X_{i} \cap \operatorname{ker} N=\{0\}
$$

and hence we find

$$
\mathcal{S}^{2}=\operatorname{im} M_{i}+\operatorname{im} X_{i}=\mathcal{S}^{1},
$$

and the algorithm stops. In the second case we have

$$
\operatorname{im} X_{i} \subseteq \operatorname{ker} N,
$$

which further implies that

$$
\operatorname{im} X_{i} \cap \operatorname{ker} N=\operatorname{im} X_{i} .
$$

Therefore, we obtain

$$
\mathcal{S}^{2}=\operatorname{im} M_{i}+\operatorname{im} X_{i}+\operatorname{im}\left(X^{2}\right)_{i} .
$$

Since $X$ is a distance-information preserving matrix with respect to a connected graph and $\operatorname{dist}\left(i, V_{O}\right)>1$, there is vertex $j$ such that $\left(X^{2}\right)_{i, j} \neq 0$ while $M_{i, j}=0$ and $X_{i, j}=0$. Hence, $\mathcal{S}^{2}$ is strictly larger than $\mathcal{S}^{1}$, and we can continue with the algorithm. By applying the same arguments repeatedly, we obtain (8a). In addition, it follows from (9) and (10) that

$$
N M_{i}=N X_{i}=\cdots=N\left(X^{d_{i}-1}\right)_{i}=0 .
$$

Furthermore, we have

$$
N\left(X^{d_{i}}\right)_{i} \neq 0 .
$$

As such, (8b) holds.

The subspaces $\mathcal{S}_{i}^{*}$ are $(N, X)$-invariant and contain $\operatorname{im}\left(M_{i}\right)$ by definition, and so they satisfy conditions $\mathrm{C} 1$ and $\mathrm{C} 2$ of the geometric version of the fault detection and identification problem discussed in section III. The result of Lemma 5 enables us to device a purely graph-topological sufficient condition for the output separability condition C3. To formulate the graph-topological condition, we need to introduce some nomenclature.

Given a simple graph $G=(V, E)$ and a pair $\left(V_{F}, V_{O}\right)$, define

$W_{O}=\left\{j \in V_{O} \mid \operatorname{dist}(i, j)=\operatorname{dist}\left(i, V_{O}\right)\right.$ for some $\left.i \in V_{F}\right\}$.

In other words, $W_{O}$ consists of the observer vertices that are the closest to one of the faulty vertices. Now, we define the bipartite graph $G_{O F}=\left(W_{O}, V_{F}, E_{O F}\right)$ by

$$
\{j, i\} \in E_{O F} \Leftrightarrow j \in W_{O}, i \in V_{F}, \operatorname{dist}(i, j)=\operatorname{dist}\left(i, V_{O}\right) .
$$

With these preparations, we are ready to state the graphtopological sufficient condition for the output separability requirement $\mathrm{C} 3$ for the class of distance-information preserving matrices.

Theorem 6 Consider the system (5) for a simple graph $G=$ $(V, E)$ with faulty vertices $V_{F}$ and observer vertices $V_{O}$. Then the family of subspaces $\left\{\mathcal{S}_{i}\right\}_{i=1}^{q}$ is output separable for any distance-information preserving matrix $X \in \mathcal{Q}(G)$ if the bipartite graph $G_{O F}$ admits a constrained q-matching.

Proof: For a given distance-information preserving matrix $X \in \mathcal{Q}(G)$, let

$$
R=\left[\begin{array}{llll}
N\left(X^{d_{1}}\right)_{1} & N\left(X^{d_{2}}\right)_{2} & \cdots & N\left(X^{d_{q}}\right)_{q}
\end{array}\right],
$$

where $d_{i}=\operatorname{dist}\left(i, V_{O}\right)$. Lemma 5 gives us that

$$
N \mathcal{S}_{i}^{*}=\operatorname{im} N\left(X^{d_{i}}\right)_{i},
$$

which implies that the output separability of the subspaces $\left\{\mathcal{S}_{i}^{*}\right\}_{i=1}^{q}$ is equivalent to the condition that

$$
\operatorname{rank} R=q \text {. }
$$

Observe that the $j$ th entry of the column vector $N\left(X^{d_{i}}\right)_{i}$ is nonzero if and only if $\operatorname{dist}(i, j)=d_{i}=\operatorname{dist}\left(i, V_{O}\right)$. Furthermore, note that the vertices in $W_{O}$ correspond to the nonzero rows of the matrix $R$. This means that the matrix obtained from $R$ by discarding the zero rows belongs to the pattern class $\mathcal{P}\left(G_{O F}\right)$. Since the bipartite graph $G_{O F}$ admits a constrained $q$-matching, it follows from Theorem 2 that $\operatorname{rank} R=q$. Consequently, the family $\left\{\mathcal{S}_{i}^{*}\right\}_{i=1}^{q}$ is output separable. 


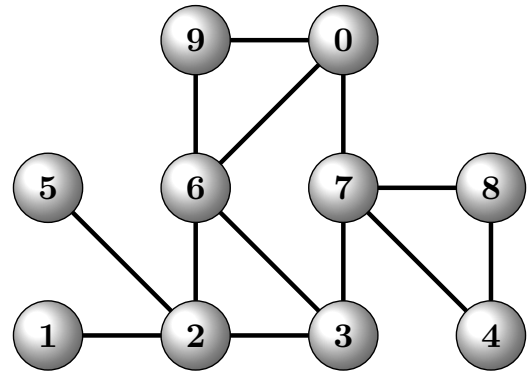

Fig. 1. A 10-vertex graph

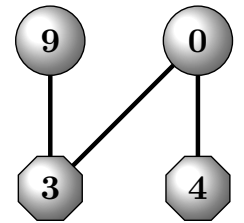

(a) $V_{F}=\{0,9\}$ and $V_{O}=\{3,4\}$

Fig. 2. Two different choices of observer vertices
Next we illustrate the result of Theorem 6 by means of an example.

Example 7 Consider the graph depicted in Figure 1. First, we choose $V_{F}=\{0,9\}$ and $V_{O}=\{3,4\}$. Note that $\operatorname{dist}\left(0, V_{O}\right)=\operatorname{dist}\left(9, V_{O}\right)=2$ and $W_{O}=\{3,4\}$. For this case, the bipartite graph $G_{O F}$ is depicted in Figure 2(a) in which the observer vertices are indicated by octagons. The 2-matching $\{\{0,4\},\{9,3\}\}$ is a constrained matching. As such, Theorem 6 concludes that the output separability condition $\mathrm{C} 3$ holds for this case, for any distance-information preserving matrix $X \in \mathcal{Q}(G)$.

Now, take the same graph and faulty vertices but change the set of observer vertices to $V_{O}=\{2,3\}$. Note that $\operatorname{dist}\left(0, V_{O}\right)=\operatorname{dist}\left(9, V_{O}\right)=2$ and $W_{O}=\{2,3\}$. The new bipartite graph $G_{O F}$ is depicted in Figure 2(b). There are two 2-matchings, namely $\{\{0,3\},\{9,2\}\}$ and $\{\{0,4\},\{9,3\}\}$. As such, neither of them is a constrained matching and we cannot conclude whether the output separability condition is satisfied by employing Theorem 6.

The result of Theorem 6 can also be used for the design of systems on graphs: given a set of faulty vertices $V_{F}$, one way to guarantee output separability is to place sensors at certain (non-faulty) observer vertices so that the matching condition of Theorem 6 is satisfied.

The next example illustrates a pathological case for which the matching condition of Theorem 6 is not satisfied for any choice of observer vertices.

Example 8 Consider the graph depicted in Figure 1. Suppose that $V_{F}=\{1,5\}$. Since $\operatorname{dist}(1, i)=\operatorname{dist}(5, i)$ for any vertex $i \notin V_{F}$, there is no choice of $V_{O}$ for which the matching condition of Theorem 6 can be satisfied.

Based on this example, we can prove the following lemma, which can be seen as a necessary condition for output separability for the class of distance-information preserving matrices.

Lemma 9 Consider the system (5), and let $A$ denote the adjacency matrix corresponding to the simple graph $G=$ $(V, E)$. Let $\{i, j\} \subseteq V_{F}$. Suppose that $\operatorname{dist}(i, k)=\operatorname{dist}(j, k)$ for any $k \notin\{i, j\}$. Then for the choice of $X=A$, the subspaces $N \mathcal{S}_{i}^{*}$ and $N \mathcal{S}_{j}^{*}$ coincide.

Proof: It can be verified that $A$ is a distanceinformation preserving matrix with respect to the graph $G$. As $\operatorname{dist}(i, k)=\operatorname{dist}(j, k)$ for any $k \notin\{i, j\}$, we have that $A_{i, k}=A_{j, k}$ for any $k \notin\{i, j\}$. Moreover, we have

$$
\left(A^{\ell}\right)_{i, k}=\left(A^{\ell}\right)_{j, k}
$$

for any integer $\ell \geqslant 1$ and $k \notin\{i, j\}$, which implies that

$$
N\left(A^{\ell}\right)_{i}=N\left(A^{\ell}\right)_{j}
$$

for all $\ell \geqslant 1$. Then, Lemma 5 implies that

$$
N \mathcal{S}_{i}^{*}=N \mathcal{S}_{j}^{*} .
$$

As such, the output separability requirement is not satisfied by the choice of $X=A$.

\section{CONCLUSions}

In this paper we have studied the fault detection and isolation problem for systems defined over graphs. First, we have provided a characterization of the so-called conditioned invariant subspaces of such systems with the distanceinformation preservation property. These subspaces play a major role in the analysis of fault detection as well as design of fault detectors. Based on this characterization, we have presented graph topological sufficient conditions for the socalled output separability requirement that is the crux of the fault detection problem in the setting of geometric control. The graph topological sufficient condition was illustrated on two examples. Based on another example, we have also presented a condition under which the output separability fails for the class of distance-information preserving matrices.

Investigating sharper necessary conditions, devising observer vertex selection methods and formulating conditions that would guarantee the asymptotic stability of fault detectors are among the future research problems.

\section{REFERENCES}

[1] G. Basile and G. Marro. Controlled and Conditioned Invariants in Linear System Theory. Prentice Hall, 1992.

[2] D. Hershkowitz and H. Schneider. Ranks of zero patterns and sign patterns. Linear and Multilinear Algebra, 34:3-19, 1993.

[3] S. Jafari, A. Ajorlou, and A.G. Aghdam. Leader localization in multi-agent systems subject to failure: A graph-theoretic approach. Automatica, 47(8):1744 - 1750, 2011.

[4] M.-A. Massoumnia. A geometric approach to failure detection and identification in linear systems. PhD thesis, MIT, 1986. 
[5] M.-A. Massoumnia. A geometric approach to the synthesis of failure detection filters. IEEE Trans. Aut. Contr., 31(9):839-846, 1986.

[6] F. Pasqualetti, A. Bicchi, and F. Bullo. Consensus computation in unreliable networks: A system theoretic approach. IEEE Trans. on Automatic Control, 57:90-104, 2012.

[7] M.A. Rahimian and A.G. Aghdam. Structural controllability of multiagent networks: Robustness against simultaneous failures. Automatica, 49(11):3149 - 3157, 2013.

[8] H.L. Trentelman, A.A. Stoorvogel, and M. Hautus. Control Theory for Linear Systems. Communications and Control Engineering Series. Springer-Verlag, London, 2001. 